\title{
Informes
}

\section{PROGRAMA LATINOAMERICANO DE ACTIVIDADES EN POBLACIÓN}

El Programa Latinoamericano de Actividades en Población (PROLAP) quedó formalmente institucionalizado como un programa especial del Consejo Latinoamericano de Ciencias Sociales (CLACSO) durante la XIII Asamblea Ordinaria de CLACSO que se llevó a cabo del 3 al 6 de diciembre de 1985 en Montevideo, Uruguay. Culmina así una larga etapa de reflexión y actividades que tuvieron como finalidad preparar el camino para este nuevo programa de actividades, a nivel regional, en los campos de investigación, docencia y comunicación en materia de población.

\section{Antecedentes}

El PROLAP es la continuación de una rica experiencia que los científicos sociales y las instituciones interesadas en el campo de la población han venido acumulando en el terreno de la colaboración regional en materia de reflexión, investigación y enseñanza de la demografía en la región. Como foro de discusión e intercambio académico a nivel regional se creó en 1968 la Comisión de Demografía, reformulada en 1971 como Comisión de Población y Desarrollo, en el seno de CLACSO. La Comisión de Población y Desarrollo llevó a cabo sus actividades mediante la constitución de grupos de trabajo sobre áreas específicas. Por orden de antigüedad, los tres grupos de trabajo que integraron la Comisión se concentraron en las siguientes áreas: migraciones, reproducción de la población y sistema de información sociodemográfica.

En 1973 se creó el Programa de Investigaciones Sociales sobre Población en América Latina (PISPAL) ${ }^{1}$ con el objetivo general de orientar y promover la realización de investigaciones sociales sobre las relaciones entre población y desarrollo, tendientes a asentar la base científica que permitiera incorporar de manera explícita la población en la formulación y ejecución de las políticas globales, sectoriales y regionales de desarrollo económico y social, así como evaluar las consecuencias poblacionales que de dichas políticas pudieran derivarse. El PISPAL fue la vía para canali-

1 PISPAl se ubica como otro grupo de trabajo de la Comisión de Población y Desarrollo de 1973 a 1977 . 
zar fondos que permitieran ampliar la capacidad de investigación de los centros nacionales, y promover la incorporación y el adiestramiento de investigadores noveles en el campo de población.

Los avances y cambios ocurridos desde esos años en el conocimiento y en los fenómenos demográficos mismos han sido significativos en la región. Sin embargo, a pesar de lo anterior existe una creciente demanda de información y conocimientos en materia de población, formación de recursos humanos de elevada calificación, y formulación, realización y evaluación de las políticas poblacionales. Además, el desarrollo institucional es aún desigual e insuficiente entre los países de la región. ${ }^{2}$

Dentro de este marco general de evolución de las experiencias de intercambios interinstitucionales en la región debe contemplarse el hecho de la terminación del PISPAL en 1985 y la iniciativa surgida en el seno de la Comisión de Población y Desarrollo de modificar su forma de operar. La confluencia de las circunstancias anteriores sugería a la comunidad de centros y a los científicos sociales con intereses en el área de población la conveniencia de formular un nuevo programa de actividades a nivel regional, como un programa de CLACSO.

\section{Objetivos, líneas de actividad y aspectos organizativos del PROLAP}

A continuación se resumen brevemente las principales conclusiones que resultaron del Encuentro Latinoamericano de Centros con Actividades en Población, realizado entre el 16 y el 21 de septiembre de 1985 en Campinas, São Paulo, Brasil. Este encuentro fue convocado y organizado por el Comité ad hoc de Transición para el establecimiento del PROLAP con el objetivo de reunir a representantes de centros latinoamericanos con actividades en población (se contó con la representación de 49 centros) para discutir y definir las áreas que el PROLAP debería privilegiar, así como sus contenidos sustantivos, mediante una propuesta concreta de actividades para los primeros dos años de existencia del programa. Con este encuentro culminaba una serie de actividades preparatorias llevadas a cabo por el propio Comité ad hoc de Transición y, con anterioridad, por la Comisión de Propuesta para el establecimiento de un programa regional en el campo de población.

El objetivo general de PROLAP es el fortalecimiento de una red institucional de centros de investigación y de docencia en población a fin de ampliar la capacidad para formar recursos humanos, realizar investigaciones sobre los fenómenos de población, promover el intercambio de experiencias y la difusión y comunicación de resultados dentro de la región,

${ }^{2}$ Véase al respecto, Daniel Rodríguez, Evolución y situación actual de las instituciones que realizan actividades académicas en población y desarrollo en América Latina, PISPAL, México, 1984. 
y estrechar los lazos de interacción con la comunidad académica internacional. El PROLAP pretende también constituirse como punto de referencia e impulsar una perspectiva latinoamericana sobre las cuestiones de población.

A nivel de objetivos especificos el PROLAP se orienta a:

i) apoyar en general la consolidación de las instituciones y, en particular, la de las de menor desarrollo relativo y las que puedan encontrarse en situaciones de emergencia; ii) incentivar el entrenamiento de investigadores y la creación de grupos nacionales de investigación en aquellos países donde no existen; y iii) asegurar en la región un flujo permanente de información sobre las actividades de investigación y docencia en el campo de la población.

Las líneas de actividad del PROLAP se desprenden de los objetivos arriba señalados. Para cumplir con dichos objetivos, el PROLAP se propone desarrollar actividades de promoción y coordinación de la investigación, la docencia y la comunicación, que tengan como referencia la situación particular de los centros en su contexto nacional, así como las actividades de conjunto que se diseñen en términos subregionales y regionales.

En consecuencia, el PROLAP se conforma sustantivamente alrededor de tres áreas -investigación, docencia y comunicación- y se propone llevar a cabo actividades con énfasis nacional, regional y subregional.

Entre los principios que rigen la organización del PROLAP se cuentan los de afiliación libre, iniciativa responsable, solidaridad, coordinación flexible y descentralización.

El PROLAP se organiza bajo la forma de una comisión de CLACSO y se integra plenamente a la organización de éste. Desde el punto de vista de su organización interna, el PROLAP contará con un Consejo de Centros y con un Comité de Coordinación integrado por un coordinador general, tres coordinadores de área - uno para el área de investigación; uno para el área de docencia y uno para el área de comunicación-y un representante del Consejo de Centros.

El Consejo de Centros es el organismo máximo interno del PROLAP y se integra por todos los centros miembros del mismo. El Comité de Coordinación es el organismo de ejecución de las actividades del programa. Todos los miembros del Comité serán elegidos por el Consejo de Centros y durarán dos años en sus funciones, pudiendo ser reelectos por una sola vez. El coordinador general será el encargado de relacionar el trabajo de los tres coordinadores de área, presidirá el Comité de Coordinación, servirá de nexo con la Secretaría Ejecutiva de CLACSO y representará al PROLAP ante terceros. Los coordinadores de área serán los responsables de cumplir el plan de actividades aprobado y de impulsar y coordinar las tareas respectivas en cada una de sus áreas; serán elegidos en la doble condición de académicos a título de sus cualidades personales y de miembros integrantes de un centro. 
Los coordinadores de área, el coordinador general y el representante del Consejo de Centros deberán pertenecer a centros distintos, procurándose además una representatividad subregional entre ellos. Los coordinadores de área deberán a su vez invitar a un centro de cada una de las subregiones, distintas de aquélla a la que pertenecen, para actuar como colaboradores en la formulación y ejecución del programa bajo su responsabilidad. ${ }^{3}$

Para el primer periodo de funcionamiento, el directorio del PROLAP quedó constituido de la siguiente manera:

Coordinador general: Alfredo Lattes, CENEP, Argentina (designado en el $39^{\circ}$ periodo de sesiones del Comité Directivo de CLACSO).

Coordinación de investigación: Daniel Hogan, NEPO, Brasil.

Coordinación de docencia: Susana Lerner, CEDDU, México.

Coordinación de comunicación: José Blanes, CERES, Bolivia.

Representante de centros: Allan Lavell, CSUCA, Centroamérica.

Francisco Alba

${ }^{3}$ Para mayores detalles sobre objetivos, líneas de actividad y aspectos organizativos véase el documento "Programa Latinoamericano de Actividades en Población (PROLAP)" preparado por el Comité ad hoc de Transición con base en la propuesta de Programa (PROLAP/Doc. núm. 1) y las conclusiones del Encuentro Latinoamericano de Centros con Actividades en Población. Este documento cuenta además con dos anexos: uno que describe brevemente las funciones de los Coordinadores del PROLAP y otro que informa sobre el Encuentro arriba mencionado. 